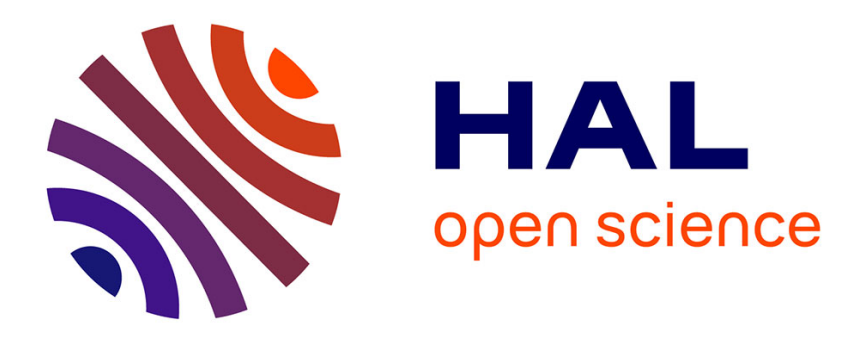

\title{
Onset of macroscopic deformation. Microscopic processes (Moderator's Comment)
}

\author{
S. Takeuchi
}

\section{To cite this version:}

S. Takeuchi. Onset of macroscopic deformation. Microscopic processes (Moderator's Comment). Revue de Physique Appliquée, 1988, 23 (4), pp.405-408. 10.1051/rphysap:01988002304040500 . jpa00245788

\section{HAL Id: jpa-00245788 https://hal.science/jpa-00245788}

Submitted on 1 Jan 1988

HAL is a multi-disciplinary open access archive for the deposit and dissemination of scientific research documents, whether they are published or not. The documents may come from teaching and research institutions in France or abroad, or from public or private research centers.
L'archive ouverte pluridisciplinaire HAL, est destinée au dépôt et à la diffusion de documents scientifiques de niveau recherche, publiés ou non, émanant des établissements d'enseignement et de recherche français ou étrangers, des laboratoires publics ou privés. 


\title{
Onset of macroscopic deformation. Microscopic processes (Moderator's Comment)
}

\section{S. Takeuchi}

\author{
Institute for Solid State Physics, University of Tokyo, Roppongi, Minato-ku, Tokyo 106, \\ Japan
}

\section{SCOPE}

In this session, we set the problem of plasticity for the following simplest situation: (1) single-phase, single crystals; (2) deformation in simple tension or compression; (3) the low temperature regime, i.e. atomic diffusion is not concerned with the plasticity; (4) the initial stage of plastic deformation.

The most important aim of materials scientists in the plasticity field is to be able to predict the plastic behavior of a particular crystal in terms of various materials parameters such as the composition, grain-size, etc. and of deformation conditions such as the temperature and the strain-rate, i.e. to describe the constitutive equation of plasticity. Even in the above most simplified situation, however, we are still far behind the goal of our aim. In this note, we try to summarize the problems of the microscopic mechanisms that control the macroscopic yielding process in the above simple situation; to solve them is essential to our goal.

\section{QUESTIONS}

The main questions to be answered would be:

(1) How does the activity of dislocation glide develop by stressing?

(2) How does the macroscopic yielding take place by creating the quasi-steady state of dislocation activity?

(3) At what conditions does the fracture replace the yielding?

In our session, Hirsch et al. has treated the third problem. They investigated the ductile-brittle transition (DBT) in silicon single crystals by introducing a well-defined crack. They showed that the ductility of the crystal is determined by competing processes of increasing stress-concentration at the crack-tip by increasing applied stress and the shielding process of the stress-concentration by dislocations multiplied from nearby sources. As a result, the strain-rate dependence of the DBT temperature obeys the same activation process as the dislocation mobility. Kratochvil, on the other hand, has developed a theory of the dislocation pattern formation in the initial stage of plastic deformation (stage I). He showed that a stress-gradient induces a non-uniform distribution of plus- and minus-sign edgedipoles initially distributed uniformly, and argued the mechanism by which the nonuniform distribution causes the enhancement of the stress-concentration, leading to instability of the uniform distribution of the dipoles. He solved kinematical equations for the formation of dipole clusters.

These two papers are concerned with the processes before and after the general yielding. In the following, we will discuss briefly what factors determine the macroscopic yielding and which microscopic mechanisms are yet to be elucidated in the future.

\section{TWO TYPES OF YIELDING}

For any finite motion of dislocations, the crystal undergoes a plastic deformation. However, the macroscopic yielding can be defined by the state where the plastic deformation rate becomes comparable with the applied strain-rate. At this stage the dislocation activities are generally in a quasi-steady state corresponding 
to the strain-rate.

To continue plastic deformation, glide dislocations must be continuously multiplied. Whatever the dislocation sources are, one needs a critical stress for the source operation, $\tau_{c} \cdot$ Let the shear modulus be $\mathrm{G}$ and the Burgers vector $b, \tau_{c}$ is usually expressed as $\tau_{\mathrm{Gb}} / \lambda$, where $\lambda$ is a characteristic length determined either by the Frank-Read source length or the spacing between strong obstacles on the slip plane, such as precipitates, forest dislocations, etc. On the other hand, the glide dislocation has its own mobility determined by some friction mechanism. Thus, the mean dislocation velocity, $\bar{v}$, averaged over the iffe of a multiplied dislocation, can be schematically drawn, as a function of the applied stress $\tau_{a}$, by thick lines in the figure: for $\tau_{a}<\tau_{c}, \bar{v}=0$ and for $\tau_{a}>\tau_{c}$, $\bar{v}$ tends to the mobility of a straight dislocation. In yielding process under a constant strain-rate test, in order to produce an enough plastic strain-rate to follow the applied strain-rate, each multiplied dislocation must attain an enough mean velocity $\bar{v}_{\dot{\varepsilon}}$.

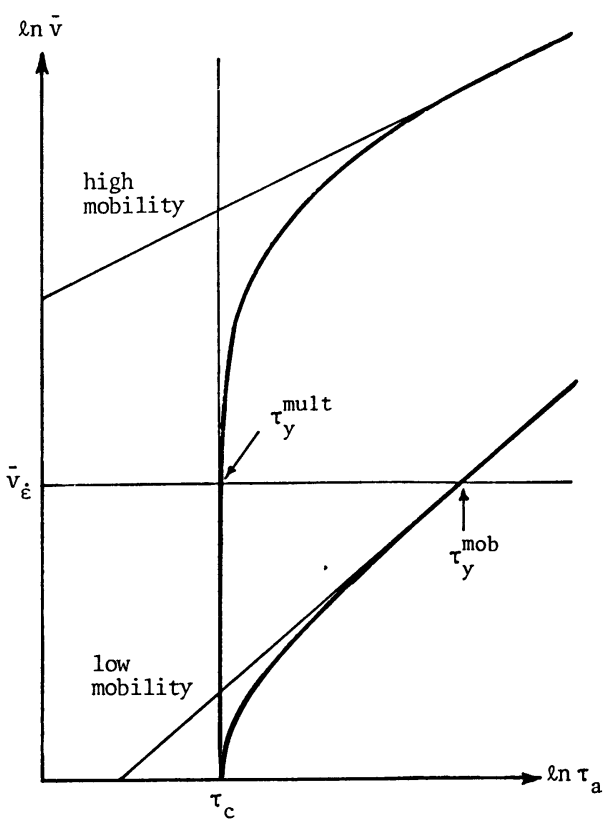

There are two types of yielding: one is the multiplication-controlled yielding for high mobility case and the other is the mobility-controlled yielding for low mobility case. In the former case, $\bar{v}$ can attain $\bar{v}_{\dot{\varepsilon}}$ value at a stress only slightly

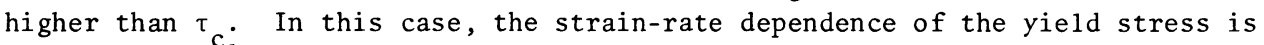
weak, i.e. $\tau_{y}^{m u l t} \sim \tau_{c}$. Meanwhile, in the latter case the velocity of dislocations at a stress near $\tau_{c}$ is so low that for the $\bar{v}$ to attain $\bar{v}_{\dot{\varepsilon}}$ value a much higher stress than $\tau_{c}$ is required, i.e. $\tau_{y}^{\text {mob }} \gg \tau_{c}$. In such a case the strain-rate dependence of the yield stress directly reflects the stress-dependence of the dislocation velocity

PROBLEMS - solved and unsolved-

According to the above argument on yielding process, microscopic mechanisms that control the yielding process are: (1) mechanisms that govern the mobility of individual dislocations; (2) mechanisms that govern $\tau_{c}$; (3) mechanisms that determine $\bar{v}_{\dot{\varepsilon}}$, or equivalently the mobile dislocation density at the macroscopic yielding stage, which is related to development of a quasi-steady state.

(1) Dislocation Mobility

The dislocation mobility is determined either by (a) the Peierls potential, (b) the pseudo-Peierls mechanism, (c) interaction with solute atoms or (d) friction due to scattering of phonons and electrons.

Peierls potentials in crystals with the bcc structure and in alkali-halide crystals are now rather well understood, and the dislocation motion in these crystals, at low temperatures, has been analysed based on the smooth-kink mode1, though the experiment and the theory are not always compatible in details. Covalent crystals with the diamond structure are considered to be the typical material in which the dislocation motion is governed by the Peierls mechanism. The dislocation mobility in those crystals has usually been analysed in terms of the abrupt kink model and the kink diffusion theory. However, relative height of the Peierls potential of the second kind and the role of it in the dislocation mobility are not yet clear.

The fact that the dislocations in crystals with a complex crystal structure have almost unexceptionally a low mobility, is due to the inherent high Peierls potential owing to the inevitable small d/b value in those crystals (d: 1 attice spacing of the glide plane), as the classical Peierls-Nabarro theory on the Peierls 
stress indicates. Besides the Peierls-Nabarro expression of the Peierls stress obtained based on a simple model, we have no general description of the Peierls stress, and also it is difficult to experimentally estimate a high value of the Peierls stress because the fracture sets in at low temperature, and thus the understanding of the mobility of dislocations controlled by the Peierls mechanism is still quite limited.

A typical case where the dislocation motion is governed by the pseudo-Peierls mechanism is the prismatic slip $(\vec{b}=\vec{a})$ in hcp crystals. In compound crystals, especially in ordered crystals, the dislocation with a large Burgers vector tends to dissociate often in a complex fashion, which can lead to the pseudo-Peierls mechanism of dislocation motion. It is, however, generally not easy to separate the two contributions to the dislocation mobility, i.e. Peierls potential and constriction energy of a non-planar spreading of the core.

Dislocation in fcc crystals and basal dislocations in hcp crystals are believed to have negligibly small Peierls stress (though the interpretation of Bordoni internal friction peaks is not consistent with this issue), and the mobility is determined by the mechanisms (c) and (d) above. The mobility of dislocations governed by solidsolution hardening has long been treated theoretically by many researchers, but experimental information about it is stil1 very poor. Quantitative comparison between the theory and the experiment on the dislocation mobility in solid-solution alloys has scarcely been performed successfully. The difficulty of this kind of experiment may lie in the fact that yielding of the fcc solid-solutions is generally not mobility-controlled but multiplication-controlled. The solid-solution hardening is an old problem, but more experimental efforts seem necessary in quantitative evaluation of dislocation mobility in solid-solutions and its relation to yielding.

\section{(2) $\tau_{c}$}

$\tau_{c}$, the critical multiplication stress, is most easily and accurately estimable in case where it is determined by precipitates or dispersing particles. In other cases, however, it is not easy to identify the mechanism that determines $\tau_{c}$, because the nature of the multiplication source in a crystal is hardly understandable. The reason of this difficulty comes from the fact that the multiplication process in a crystal is, so to say, an intermediate range process which is beyond the range of the TEM scale; we have almost no appropriate technique to identify the real multiplication process during the yielding process. On the other hand, whereas the dislocation mobility varies over many orders of magnitude from crystal to crystal, the multiplication stress, if not determined by precipitates, would be of a similar order of magnitude among crystals, because the multiplication process may depend on the dislocation structure which is more or less similar among crystals. Very roughly, $\tau_{c}$ will be of the order of $10^{-4} \mathrm{G}$. Therefore, it may be generally stated that the yielding of a crystal (again if not precipitation-hardened) is multiplication-controlled only when the dislocaiton mobility at the testing temperature is low enough. Thus, the yielding in soft metals such as fcc metals and their dilute solid-solution alloys, and ionic crystals, bcc metals and covalent crystals at enough high temperatures are multiplication-controlled.

So far, the $\tau_{c}$ has been assumed to be determined athermally. If the dislocation source is locked by an atmosphere, the operation of the source is thermallyactivated. It is, however, usually believed that new fresh sources can be produced during deformation, e.g. by cross-slip. Yet, it is not clear if this is always possible even for widely dissociated dislocations such as those in some fcc alloys. Another special case is, so to speak, a self-trapping of multiplying dislocations by the transition from glissile to sessile configuration. A famous case is <110> $\{111\}$ dislocation in $\mathrm{L}_{2}$ compounds such as $\mathrm{Ni}_{3} \mathrm{Al}$, known as the Kear-Wilsdorf locking. In such a case, the source activation can only be achieved under the condition that the velocity of glide dislocation is so fast as not to be trapped by a thermallyactivated transition from glissile to sessile. The yielding of such crystals is multiplication-controlled; the strain-rate dependence of the yield stress in $\mathrm{Ll}_{2}$ compounds is in fact very small.

(3) Quasi-steady State at Yielding

In the mobility controlled yielding, the yield stress depends on the $\bar{v}_{\dot{\varepsilon}}$ value, or in other words on the moving dislocation density at yielding stage. The phenomenological theory of mobility-controlled yielding has earlier been established, which is known as the Johnston-Gilman theory. In the original theory, the mobile dislocation density has been assumed to be a monotonically increasing function of strain. Later, however, it has been analysed experimentally that a quasi-steady state 
continues during the stage $I$, where the mobile dislocation density is kept almost constant. The mobile dislocation density $\rho$ at the quasi-steady state is usually proportional to $\tau^{2}$, and hence $\dot{\varepsilon} \equiv \rho b \bar{v} \propto \tau^{\mathrm{m}+2}$, where $\mathrm{m}$ is the stress exponent in the dislocation mobility equation. The quasi-steady state is realized by an equilibrium state between the rate of the multiplication of mobile dislocations and the rate of the decrease of them by mutual annihilation or immobilization by dipole formation. However, detailed mechanism of the realization of the steady-state have not been well understood, mainly hecause of the lack of the knowledge about the nature of the multiplication sources.

\section{FINAL REMARKS}

As mentioned so far, the solved problems are still quite limited compared with the unsolved problems. Basically, the difficulty in the physics of plasticity consists in the fact that the dislocation behavior is of strong individuality; it varies sensitively depending on the detailed dislocation core structure and the bonding nature of the crystal, even the crystal structure is the same. Also, the dislocation processes that produce plasticity are somewhat in between macroscopic and microscopic in nature, in the sense that the plasticity cannot be described only by single-dislocation process nor simply by statistical averaging procedure of a group of dislocations. Very often, an important role seems to be played by dislocation processes in our blind point of the observation: the dislocation processes of an intermediate range between the TEM scale and the X-ray resolution scale. Nevertheless, we believe that our continuous efforts toward better understanding of the plasticity problems by ingenious experimental ideas and theories, as well as simulation studies, will steadily bring us to approach our final goal. 